\title{
The Spread of League of Legends
}

\author{
Yile Liu ${ }^{1, *}, \dagger$, Yitao $\mathrm{Ma}^{2, \dagger}$, Tianhui Wang ${ }^{3, \dagger}$
}

\author{
${ }^{1}$ Kimball Union Academy Meriden, New Hampshire 03770 USA \\ ${ }^{2}$ Guangyi International Academy, Changsha, Hunan, 410000, CN \\ ${ }^{3}$ Maple Leaves International School, Nanjing Jiangsu, 21000, CN \\ *Corresponding author: guanghua.ren@gecacdemy.cn \\ ${ }^{\dagger}$ These authors contributed equally
}

\begin{abstract}
League of legends is the most famous online video game around the word. The game is created by Riot Games from USA in 2009. It is a type of Multiplayer Online Battle Arena games, 2 groups and combats with 5 players each group with impressive 3D graphics. In a fast developed society with so many entertainments, the League of Legends is represented as a the most successful one in the e-sport industry. The purpose of this study was to explore how did advertising promote the spreading of games from the game to the players. In order to find out the answer, this paper first reviewed the theory of consumer loyalty, customer satisfaction as well as the word of mouth. According to the theory of the subject mentioned above, it is suitable for League of Legend. Thus, this paper makes the hypothesis that League of Legend uses customer and peripheral products to promote the spread of their products and makes a discussion based on the analysed result.
\end{abstract}

Keywords: Consumer Loyalty, Word of Mouth, League of Legend

\section{INTRODUCTION}

\subsection{Background}

The Multiplayer online battle arena (MOBA) is a potential branch in today's video game industry. MOBA game divides players into different lanes and makes the players cooperate to win the game. Of all the other MOBA games on the market, LOL (League of Legends) earns the most popularity. It has an enormous player population and the most large-scale international competition globally. The number of players is still increasing from 2009, which is the year this game establishes. The game was invented by Riot Games and stepped into China in 2011 through the agency Tencent [2]. According to the League feed website, the average monthly player was approximately $115,000,000$ this October. LOL' s popularity shows an upward trend since it's entering the Asian market, and the story of famous players such as Uzi, Faker, and The Shy was eulogized among players. The game's popularity in China peaked when Invictus Gaming (IG) won the 2018 world championship, a cup that Chinese players have been craving for six years. From 2018 to now, more and more capitals have entered this trend. Though this might form some parents' opposition, League of Legend and the industrial chain behind it has stabilized.

LOL has operated for over ten years, but still, new games are joining this game. This is a situation that almost no games before had occurred, and many scholars did dig deep into the effect of the game. On the Chinese "Zhiwang" Website, a website that contains the most authoritative student papers, over 1000 papers include some topics about the LOL. Some of the papers include topics comparing LOL to other games. Some of the papers contain subjects about how the game's commercialization works and discuss The CompanyRiot Game more deeply. Most video games have only two and three years to be famous and hot. Nevertheless, LOL manages to keep that for ten years, and the reason for this is what this research plan will focus on.

From the team's experience, the group believes LOL is an excellent example of how a company should commercialize a game. Not only the new equipment in the game, the design team progressed, but also the Peripheral products such as the cartoon "Arcane" and well-known songs such as "RISE Phoenix" Riot Games produced [1]. After releasing the first three episodes of Arcane, the Average Tomato meter on Rotten Tomatoes is now $100 \%$. The perfection of the balancing system 
makes the players have a similar game experience, igniting their spirit of cooperating. Highly publicized events bring hot topics for the game. Debatable and celebrated players are abundant.

The group believes that the success of LOL is not an accident but a phenom that is very worth discussing. The group decided to make a research paper and discuss why this game has become so popular.

\subsection{Research Gap}

In this research paper, the central research question is how LOL spreads among society. The bullet points mentioned in the introduction were the reason that the group concluded about why choosing LOL as a representative of video games and game spread is an interesting topic, and they will be discussed more in further statements. The group believes understanding the success of LOL would help people to have a more precise recognition of how a particular engaging project spread among human society. Determining how to make a product popular is essential for the group since the group comprises students who are willing to go on the road in communication major, which involves much advertising.

The group noticed that before this project, there were already papers discussing different advantages associated with LOL. These papers approach the topic with different perspectives. Some of them associate League of Legends with E-sport, while others connect them with the game industry. There was even a comparison about computer games with phone games such as "Area of Valor." Gaining experience and opinions from these papers, the group decided to assume how League of Legends became famous worldwide. The hypothesis the group finally agreed on is the topic that "LOL uses customer and peripheral products to promote the spread of their products".

\subsection{Paper Structure}

This paper is a neatly designed research paper on how LOL is a game spread among society. A research-based discussion on Consumer Loyalty and Word of Mouth will be presented in the report. The group decided to hand out questionnaires and sum up a conclusion from the answers. In the end, a conclusion will associate the hypothesis and give the future path for our research.

\section{LITERATURE REVIEW}

\subsection{Consumer Loyalty}

Consumer Loyalty (CL) was built on the foundation of the Corporate Identity (CI) which was widespread in the 1970s and the Customer Satisfaction (CS) created in 1980s. It means enterprises should use the satisfactory of demand and expectation from consumers as the target, eliminate and prevent the complaint of consumers effectively [3]. Increase the satisfaction of clients and build a kind of trustworthy relationship between the company and client. This kind of relationship can be called a Quality value chain" [4].

Oliver 1999 defined CL as a high level of commitment to consistently repeat purchases of preferred products or services in the future, resulting in repeated purchases of the same brand or series of products or services under the same brand, and without diversion due to changes in market dynamics and the attraction of competitive product marketing efforts [5]. That has become the most common explanation of consumer loyalty nowadays. CL is used in modern marketing in multiple markets. It has effects on output, sales, market occupation, profits etc.

There are five main elements of consumer loyalty: quality of products, price level, simplification of consumption process, physical and mental experience and enterprise innovation ability.

The first one is quality of products. Quality of products is the basis of rising consumer loyalty and carrying out services of high grade [5]. In some theory, the loyalty of consumers to the company or trademark can also be known as loyalty to the quality of products. Only the high-quality products can really create trust between clients and companies.

The second one is the price level. Pricing products with appropriation is also a significant method to improve and stabilize consumer loyalty [5]. Companies should avoid behaviours like short term high profits, locate the price level by regular profit and manage to reach the "price expectation" of consumers. The "price expectation" is according to the valuation standard of consumers. If the price level is over the "price expectation", then the consumers will consider that the price level is too high, the product is unworthy of one's name and the desire of purchase will decline. Meanwhile, if the product can't achieve the "price expectation", then the consumers will float doubts about the performance of the product, and hesitate on consumption.

The third one is the simplification of consumption process. Whether in a store, on a web site or in a business catalogue, the simpler the purchase process, the better [5]. Cut out all the unnecessary writing and form-filling to help the customers find the product they need, explain how it works, and do anything to simplify the transaction process, standardize the service process. In some theory, it is also named "build up a stable trench", to make sure the convenience of consumers, receive goods or services from companies, the stabilization of the trench is important [4]. Different periods of enterprise development, there must be different policies on channels, to keep channels smooth, to ensure that the win- 
win of channels and manufacturers is one of the golden laws.

The forth one is to bring customers different physical and mental experience with the unique corporate culture, if possible, establish their own standard product system and service indicators. The purpose has two aspects of significance. On the one hand, these systems and indicators can foil and render the unique personality of the enterprise, on the other hand, let the cost of switching products or services of customers increase, can construct a quite effective barrier.

The last one is enterprise innovation ability. Must establish accurate customer demand and competitive status collection and analysis system [5]. Customer demand is not invariable, especially fashion consumer goods, fast consumer goods, electronic consumer goods. It is necessary to improve the competitiveness of an enterprise to understand the changing needs of its customer groups and provide corresponding new products or services that can bring them new experiences. But at the same time, from the perspective of improving loyalty, enterprises and brands with continuous innovation are the objects of infinite vitality and longterm dependence in the mind of customers [3].

In conclusion, consumer loyalty is mainly affected by these five elements, no matter in what kind of cases. The development of the five elements is the way to improve the loyalty of consumers. About the effect of CL would have on the consumer, in order to build consumer loyalty, the influence of the game made on the consumer will be one hundred percent focused on the positive effects [4]. Like the satisfaction of consumers after consumption. This is the insurance of consumption possibilities from consumers and the construction of bonds between consumers and products.

\subsection{Word of Mouth (WOM)}

Word of mouth is commonly known as word-ofmouth marketing which use in market. WOM was originally born from the communication study. It' s first created by the past president of Nintendo, Hiroshi Yamauchi. Individual who outside the producer will through the method of express or implied, transfer of a particular or a certain types. Later, he or she will to be reminiscent of the objects of any organization or individual information. And then the person will lead the audience to get information, change the attitude, and even influence the buying behaviour of a two-way interactive communication behaviour [6].

The electronic word of mouth is formed from the traditional word of mouth, the production of this word is due to the high speed of development of international technology [7]. That makes the attitude and comments of online users is as significant as the off-online communication. To build a positive word of mouth for a firm, massive strategies are used by the producer, like detail focusing and firm story spreading [7].

Sometimes the influences to the WOM of products, is not because of the main structure of the product, the effect is actually due to some components and parts that can be ignored by the producers easily [7]. But the overlook of producer doesn't mean the neglect from the clients, the focus of these tiny details can really lead to a soar of development on word of mouth [7].

Stories are the effective tool of word of mouth spreading, because it leads the motions of human [8]. An example of "Farino" a famous brand of leather shoes manufacture. In 1189, the Ancient Roman knight Farrenaud had his soldiers cover their feet with leather to protect them from the cold during his march to Jerusalem. The last story of Farino spread among the people, build up the word of mouth.

\section{METHOD}

LOL has a lot of consumer loyalty. The group did a survey that most players had been playing the game for more than two years. LOL has a lot of great events, so it gets a lot of attention from players.

\section{1. $L O L$}

As one of the hottest e-sports games today, League of Legends has long been integrated into our lives, from professional stadiums to live broadcast platforms to our daily lives. League of Legends is developed by the American Riot Games company, which belongs to the online game of heroes versus the Multiplayer online battle arena. There are hundreds of individual heroes in the game, as well as a ranking system, a talent system, a rune system and other characteristic development systems. The League of Legends is also committed to promoting the development of global e-sports. In addition to linking the various competition areas to develop professional leagues and building an e-sports system, it also hosts three major events: "Mid-Season Championship", "Global Finals", and "All Star All-Star Game" every year. World-class competitions have won the love of hundreds of millions of players, and have formed their own unique e-sports culture [9]. Throughout the League of Legends, he is indeed a very good competitive game.

\subsection{SWOT Analysis}

\subsubsection{Strength}

LOL has a lot of advantages. First of all, the matching mechanism is very reasonable. The game will match your opponent according to the level of the game. There is not a big difference in level. Then, the content of the game is very rich. The game has already a lot of heroes, 
improving the gameplay of the game. The game is optimized in a timely manner, and bugs can be fixed in a short period of time.

\subsubsection{Weakness}

There are some downsides to the game. First, new heroes take a long time to come, and other games are updated more often than competing games [10]. Secondly, the game's win rate mechanic leaves something to be desired. After successive victories, the player will meet a higher ranked player [10]. This reduces your chances of winning. This will discourage players. Thirdly, the price of the game is high for people.

\subsubsection{Opportunity}

The group knows from the questionnaire that the game is mostly played by students. LOL has been around for more than a decade. The game is mainly played by students, mostly high school and college students. They have free time, and they have a certain level of consumption. The game was released early, and the most loyal players were in their 20s. LOL is launching a new mobile game that will expand its audience, such as middle-aged people and kids.

\subsubsection{Threaten}

LOL is under threat this year. The first is the production of the game. Now the special effects are very good, so there is little room for improvement. Second, in the early days of LOL, it led the whole Internet era, now with more and more competitive games, LOL is under constant threat even though it is difficult to improve [10].

\section{RESULT}

After more than ten years of development, LOL has become mature in all aspects, content updates quickly, and it retains a lot of players. It occupies the main game market, and its players play the game for a long time with a large consumer group. Even a mature game has its weaknesses. The position of heroes in the game is fixed, and most heroes are only suitable for one or two functions. Heroes need to be purchased and are expensive, newbies can't immediately experience the heroes they want to play, and collecting all heroes is extremely difficult [11].

\section{DISCUSSION}

Hypothesis of the group is similar to the analysis of the group. Most of the spread of the game has been through recommendations from loyal players to their friends [10]. And LOL needs to improve the quality of its games to increase player loyalty [3].
The group thinks that keeping up the pace of updates and putting more effort into making games will keep the old players coming back. LOL can increase the welfare of new players and lower the barrier for new players, which can attract more new players. The game needs to constantly update the hero's features and improve the story setting to increase the player's interest in the game. Then lower the price of the hero and make it easy and quick for beginners to enjoy the game [11].

The results of our analysis are basically the same as the results of game loyalty. This shows that players have similar criteria for satisfaction with goods. We can refer to these aspects when improving customer loyalty.

\section{CONCLUSION}

To conclude, Riot Game is a great company that can do great operations to make a game popular. The unique design ideas and high propaganda intensity make it popular all over the world. From the operation of the leagues of different countries and Championships, LOL is a game that uses these resources to make the topic of the game enduring. Also, because of adding unique game mode such as Team fight Tactics (TFT) and Ultra fire, the playability of the game is very strong. The songs Riot Game created are also ear-catching and the anime productions are striking. These are all strengths that Riot Games gain consumer loyalties. A game with high playability and mature peripheral products is always on a top recommendation if a consumer wants to recommend. This paper also demonstrates the importance of gaining consumer loyalty and worth of mouth. The group believes that a game like LOL will always have a better tomorrow, and the researchers of this paper are eager to participate in future researches on the game. The skilled use of these commercials made LOL not only a game, but an object that carries youthful memories. When Edward Gaming (EDG) scored the championship for LPL on November the 6th, how many Chinese students roared for the win. It's not as simple as a game now, but a faith from youth that carries on home country feelings.

\section{REFERENCES}

[1] Rotten, Tomatoes. (2021) Arcane: League of Legends.

https://www.rottentomatoes.com/tv/arcane_league of_legends> Accessed 1 November 2021.

[2] Spezzy. (2021) "How many people play League of Legends? - UPDATED 2021 (October)." $<$ https://leaguefeed.net/did-you-know-total-leagueof-legends-player-count-updated/ Accessed 1 November 2021.

[3] Zheyu, Liu. (2021) "Is consumer loyalty a false proposition.”. P14-16. 
[4] Sen, Zhang. (2021) "Research on the influence of corporate social responsibility on consumer loyalty." vol. 31, P2.

[5] Ruixuan, Zhang. (2021) "Correlation analysis of brand fame, identity, satisfaction and brand loyalty."2, Yanshan University NEUQ, China.

[6] Philip Kotler, Kevin Lane Keller. (2008) "Marketing Management." 13th Edition.

[7] Emanuela Villani. (2020) “A Study on social Media Elctronic Word of Mouth (ewom), Emotions and Tourists Decision Making." Zhejiang Gongshang University, China.

[8] Wanqin Shang. (2021) "Research on the expansion of communication channels under the new media environment.”. Zhengzhou University, China.

[9]] Xingyi Wang. (2019) "Explore the e-sports culture in youth subculture from the perspective of new media." vol. 12.

[10] Shunyu Yao. (2017) "Study on the Communication of e-sports Events -- Taking League of Legends as an example." P9-15. P25-27.

[11] He Yang. (2021) "Study on the influencing factors and Evaluation system of e-sports Communication effect of Network media." P26-33. 